\title{
A ROBUST POSITIONING ARCHITECTURE FOR CONSTRUCTION RESOURCES LOCALIZATION USING WIRELESS SENSOR NETWORKS
}

\author{
Meimanat Soleimanifar \\ Ming $\mathrm{Lu}$ \\ University of Alberta \\ 3-009 Markin/CNRL NREF \\ Edmonton, AB T6G2W2, CANADA \\ Ioanis Nikolaidis \\ University of Alberta \\ 3-22 Athabasca Hall \\ Edmonton, AB T6G2E8, CANADA \\ SangHyun Lee \\ University of Michigan \\ 2350 Hayward, 2340 GG Brown \\ Ann Arbor, MI 48109-2125 USA
}

\begin{abstract}
This paper introduces a cost-effective and robust positioning architecture that relies on wireless sensor networks (WSNs) for construction resources localization. The architecture determines the location of mobile sensor nodes by evaluating radio signal strengths (RSS) received by stationary sensor nodes. Only a limited quantity of reference points with known locations and pre-calibrated RSS in relation to pegs are used to lock on the most likely position coordinates of a tag. Indoor experiments were conducted, revealing that acceptable position estimation with 1-2 m accuracy can be obtained with this flexible sensor network architecture. To simulate the dynamic setting of a construction site, controlled experiments were also conducted by parking a car at various locations in the testing environment in order to evaluate the impact of imposed obstacles on location estimation performance. This localization technique is found to produce robust positioning results, thus paving the way for potential deployment in real-world construction sites.
\end{abstract}

\section{INTRODUCTION}

Emerging localization and tracking technologies enable automated data acquisition for process and project control in construction engineering (Jang and Skibniewski 2009). Effective management of construction resources, including workforce, equipment, and material, is critical to project success. Completion of project tasks on schedule, safely and within the planned budget needs a coordinated planning effort that allocates adequate availability of project resources (Teizer 2008). Successful construction projects are often determined by the level of awareness of resource status or project performance. Thus, timely information of these factors can assist in fast and confident real-time decision making.

In recent years, the need for indoor localization has also been increasing in construction sites (Khoury and Kamat 2009), presenting tremendous opportunities for research in this area. Construction tasks, including inspection and progress monitoring, need to have up-to-date access to project information in indoor or partially covered site environments, for example, tracking the steel components in fabrication shops for productivity control or localizing laborers in underground tunnel construction for the purpose of safety management. 


\section{Soleimanifar, Lu, Nikolaidis and Lee}

While outdoor localization techniques have been developed and deployed, indoor methods remain a research challenge. The Global Positioning System (GPS) is an attractive option for outdoor environments, but is not suitable for indoor applications. In addition, due to the complexity of indoor environments, unlike outdoor environments, the development of an indoor localization technique is always impeded by a set of challenges including dense multipath effect, no line-of-sight, noise interference and building material dependent propagation effects (Zhang et al. 2010). Construction environments add the further complexity of frequent changes to the environment, as materials, workers, and equipment are dynamically introduced and relocated within a site.

Due to limitation of the previously proposed approaches, recent construction research has also investigated the use of Radio Frequency (RF) technologies that measure the strength of the received signal (Luo et al. 2010). Though using RF signals is not the only option for indoor location tracking, RFbased technology has the advantage of simplicity and low cost; in particular, with the advent of low-cost wireless sensor networks, RF-based real-time positioning solutions can be easily deployed in the application environment (Haque et al. 2009).

This paper introduces a positioning architecture of wireless sensor networks that can facilitate the localization of construction resources in both indoor and outdoor environments. The introduced architecture has the potential to reduce the installation cost for the multiple sensing/positioning units that need to be installed.

Several experiments were conducted in order to examine the positioning performance, precision and robustness. We attempted evaluate the localization performance in a dynamic environment by quantifying the effects of unpredictable temporary and permanent obstacles that could appear at job sites. The proposed system architecture is briefly described and results of the indoor experiments are then presented.

\section{BACKGROUND}

Over the past decades, construction industries have showed an increasing interest in location-aware systems and services (Kim et al. 2010). The information enables construction managers to be aware of the current state of construction resources. For many years, RFID systems and the GPS have been attractive options for automated tracking and monitoring of construction assets. For resource positioning, tracking and automated data collection in construction, there is no doubt that these mature technologies are more appealing than previous technologies such as the barcode. However, several limitations have been detected in construction applications.

RFID did not meet the requirements for the harsh construction conditions as a result of inaccurate positioning based on proximity (Pradhan et al. 2009), inflexible and limited networking capabilities, and the high cost of RFID readers (Skibniewski and Jang 2009). Moreover, the communication distance between RFID tags and readers decreases significantly with the presence of metals, concrete and moisture in their vicinity which is commonplace on a building site (Lu et al. 2007).

The performance of the GPS localization system can be severely weakened due to satellite signal blockage and the multipath effect, which is caused by deflection and distortion of satellite signals in highly dense areas and temporary structures or facilities like the scaffold and formwork on a construction site (Lu et al. 2007). Due to limitations of the previously discussed technologies, the usage of WSN has been expanding in recent construction research efforts. A WSN is a self-organizing network composed of a large number of sensor nodes, closely interacting with the physical world. It features low-cost nodes, extensive network capability allowing deployment of large quantities of nodes so as to increase the network coverage, stability and reliability in wireless communication. In addition, low power consumption facilitates operation and maintenance of the system (Shen et al. 2008). Moreover, the ad-hoc network architecture makes implementation and adjustment of the network flexible.

A new tracking architecture was implemented using wireless sensor modules by combining radio frequency signals and Ultrasound; the results showed accurate position estimations with enhanced network flexibility (Skibniewski and Jang 2009). However, traditional ultrasound positioning has some disadvantages including line-of-sight transmission, multipath, high cost and power consumption which may 


\section{Soleimanifar, Lu, Nikolaidis and Lee}

hinder the possible applications in complicated construction environments (Shen et al. 2008). According to the technological functions, economics and management targets, various combinations of RFID and Zigbee-based sensor networks have also been applied for materials tracking and supply chain management (Shin et al. 2011; Cho et al. 2011). RFID tags were used to identify various kinds of construction materials, and the ZigBee communication technology was used to wirelessly transfer this information. These studies confirmed that WSN can improve the wireless communication and network flexibility but their primary use was only data transmission, and not positioning.

Considering the features of construction sites and characteristics of indoor environments, a new cost-effective and energy-efficient WSN based positioning framework is proposed that can effectively identify and track a wide range of construction resources. This framework is expected to increase reliability and robustness, which are difficult to achieve with current technologies.

\section{SYSTEM ARCHITECTURE AND POSITIONING ALGORITHM}

In terms of location estimation by wireless networks, four different measurement principles are generally implemented: received signal strength indicator (RSSI), angle of arrival (AOA), time of arrival (TOA), and time difference of arrival (TDOA) (Shen et al. 2008). AOA, TOA, and TDOA methods demand on line-of-sight communication and require expensive infrastructure. They also suffer from the presence of different materials, equipment, and building structures at construction sites. As such, Received Signal Strength Indicator (RSSI) is considered to be more suitable for localization applications on building construction sites. The majority of positioning systems employ the RSSI method owing to the fact that RSSI measurement capability is available in most wireless radio signal communication devices (Lymberopoulos et al. 2006).

Localization methods can be further classified as 1) range-based, relying on estimates of the distance or angle between the transmitter and the receiver 2) range-free, defined exclusively by the perceived connection between a tracked tag and its neighbors and 3) RSSI profiling by which the perceived characteristics of the tracked tag's signals are compared against pre-collected samples from known locations. By comparison, GPS performs triangulation based on ranges to at least four known satellites in order to fix the coordinates of the receiver, and calibrate the clock bias of the receiver (Niculescu and Nath 2001). GPS systems require expensive and energy-consuming electronics to precisely synchronize the receiver's clock with the satellite's clocks. With hardware limitations and the energy constraints of sensor network devices, the method underlying GPS and other range-based technology present a cost barrier for localization by WSN. So, solutions in range-free localization are identified as a more cost-effective alternative to the range-based approaches for large scale sensor networks (He et al. 2003). With the range-free approach, the localization problem is easy to solve, but the estimated locations tend to be crude. So, utilizing the RSSI profiling method will help to compensate for the effect of environment on the reliability of the estimated location which is obtained from perceived RF signals (Haque et al. 2009). During the profiling stage, the network collects and stores in a database (maintained on a central server) samples acquired from tags located at known points within the monitored area. The logistics of collecting such samples may involve a person moving around the area with a special variant of the tag node, e.g., equipped with a clickable map.

Our proposed localization architecture implements the RF-based localization scheme called LEMON proposed by Haque et al. (2009). This approach is based on sensing strengths of received RF signals and is a combination of a range-free method and RSSI profiling so as to take the advantages of both. This architecture is simpler and more accurate than other approaches and the uniformity and low cost of devices makes LEMON a highly viable and very practical solution for construction. The infrastructure nodes of LEMON are low-cost low-power wireless devices [EMSPCC11 by Olsonet Communications (Olsonet Communications Corp 2011)]. The node makes use of the CC1100 RF module from Texas Instruments operating within the $916 \mathrm{MHz}$ band. From an operational point of view, the node is called peg when it captures signal strength. Pegs' locations are fixed (stationary nodes) and their precise locations need not 


\section{Soleimanifar, Lu, Nikolaidis and Lee}

to be known. A monitored device, i.e., one whose location needs to be estimated, is a node of the same type as a peg, and is called a tag.

The task of location estimation in LEMON consists of two phases: profiling and actual localization. Generally, during operation in both phases, a tracked tag periodically emits RF packets. In the profiling stage, tags are located at predetermined known locations called reference points. LEMON maintains on a central repository, a database of signal strength readings from tags in relation to all the pegs. In this phase, all the pegs that can hear the RF packets emitted by the tag, will forward the data reporting the RSSI measured to the central server. The database consists of samples which are stored as tuples $\langle C ; \Omega ; \tau\rangle$ in which $C$ represents the known coordinates of the sampled point, $\Omega$ stands for the association set (which comprises peg ID and the RSSI value received by that Peg), and $\tau$ symbolizes the class of sample, identifying the RF parameters of the transmitter (such as transmission power, bit rate, and channel number). The task of actual localization of the tracked tag is exactly the same as the profiling stage. The only difference between the profiling phase and actual localization is that, in the profiling stage, the association set of tag profiling reports also include the known coordinates of the sampled point, but in the actual localization stage, the location of the tracked tags is unknown and needs to be estimated based on the location of sampled points.

In the localization stage, the server compares the perception of the tracked tag's RSSI measured by all the pegs in the monitored area against the RSSI profile of each profiled reference point and evaluates the difference between the tag and all the profiling points. If $\Omega=\left\{w_{1}, \ldots, w_{k}\right)$ and $\Psi=\left\{\psi_{1}, \ldots, \psi_{k}\right)$ are assumed to be two association's sets, the distance between these sets is:

$$
D(\Omega, \Psi)=\sqrt{\sum_{j=1}^{N}\left(R_{\Omega}(j)-R_{\Psi}(j)\right)^{2}}
$$

where $N$ is the total number of Pegs in the network and $R_{\Omega}(j)$ is defined as $r_{j}$, if the pair $<p_{j}, r_{j}>$ occurs in $\Omega$, and 0 otherwise. Therefore, the server evaluates the distance of each pre-selected sample (its association set) from the tag's association set representing the combined momentary perception of the tag's RSSI by all the pegs which can hear it. Then it selects an arbitrary number $k$ of profiled samples with the smallest distance (in terms of the $D$ metric) from the tracked tag, which is called a best matched set of profiled points. Subsequently, the coordinates of the selected samples are averaged to produce the estimated coordinates of the tag. The averaging formula biases the samples in such a way that the ones with a smaller distance (and, hence, assumed to be probably closer to the tag being localized) contribute with a proportionally larger weight. Let $D_{\max }$ be the maximum distance among the best $k$ selected samples and $S_{d}=\sum_{i=1}^{K} D_{i}$ be the sum of all those distances. The tag coordinates are estimated as:

$$
\begin{aligned}
x_{\text {est }}= & \frac{\sum_{i=1}^{k} x_{i} \times\left(D_{\max }-D_{i}\right)}{K \times D_{\max }-S_{d}} \\
y_{\text {est }}= & \frac{\sum_{i=1}^{k} y_{i} \times\left(D_{\max }-D_{i}\right)}{K \times D_{\max }-S_{d}}
\end{aligned}
$$


where $\left(x_{i}-y_{i}\right)$ are the coordinates associated with sample $i$. Note that in this approach, RSSI is only used as a numerical attribute of a profile sample whose value should be close to the perceived value. No direct attempt to associate an RSSI value with the Euclidean distance is made.

\section{SYSTEM IMPLEMENTATION AND RESULTS}

Construction sites are dynamic environments which are exposed to movement of equipment, materials and laborers. To confirm the viability and limitations of the proposed solution and to evaluate the environment variation due to the presence of an obstacle, a prototype LEMON system was assessed in an underground parking lot on the University of Alberta campus which resembles an indoor area. The absence of decorative features in the area makes it a reasonably good approximation of a structure being built. That is, the space consists of concrete floor, ceiling and pillars and metal beams to support the load of the structure. Thus, the car park could mimic the challenges and complex characteristics found on the construction site with random and continuous movement of vehicles and people. In the data collection phase, the central node is connected via a USB dongle to a laptop, where all the data collected by the network were stored and processed. During the data collection, some of the collected readings were saved in the LEMON's profile database, when some others would be stored and used as tracking data for method verification.

The experiment started by deploying a number of nodes within the monitored area. Figure 1 shows a sample distribution of nodes for the experiment. The grid was $12 \times 8 \mathrm{~m}$ (consisting of 24 ( $2 \times 2 \mathrm{~m})$ squares), in which 10 solid squares (all around the grid) were Pegs, while the 25 crosses marked with asterisks, provided profile samples whose pre-defined locations were known. The circles acted as tags whose locations were to be determined. Tags were placed in centers of the grid squares to compare their exact locations with the estimated ones so as to evaluate the accuracy of the system.

The objective of this test was to check the performance of LEMON under a traffic flow-controlled setup including four different cases: without any car, car on the right side of the monitored area, at the middle, and on the left. All the profiling points or tag locations, even those obstructed by a car, were considered. In the four "car parking" scenarios tags were located using the profiling data, which were collected at reference points only from the original setup (without any parked car in the grid) in order to identify changes in the environment.

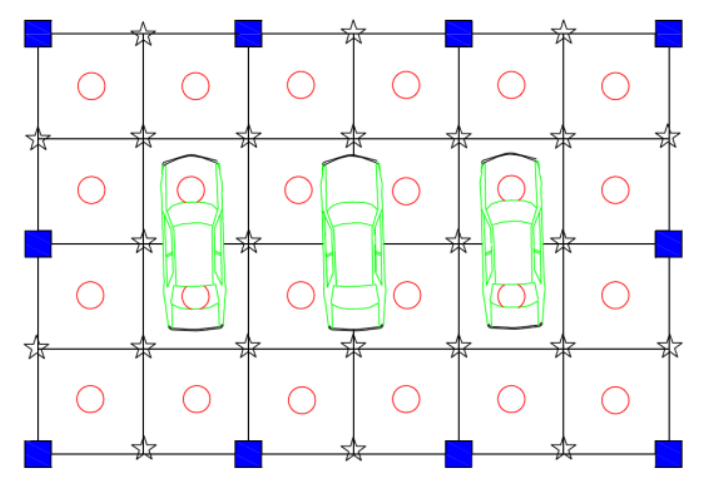

Figure 1: Experiment layout

In this experiment, the localization error magnitude is the Euclidean distance between the estimated and actual locations of a point. The average magnitude of error given different $k$ (number of bestmatched samples) and its $95 \%$ confidence level interval of the location of all the points in each case were investigated in order to find the best $k$ (Figure 2). $k=6$ is selected as it results in the smallest average localization error. Once an appropriate $k$ was decided and applied in all subsequent experiments, we turned our attention to the question of how an obstacle, which was a medium sized automobile in this 


\section{Soleimanifar, Lu, Nikolaidis and Lee}

case, could degrade the localization accuracy. The inclusion of an automobile (or any other movable metal facility) is used to mock the situations commonly encountered in construction sites.

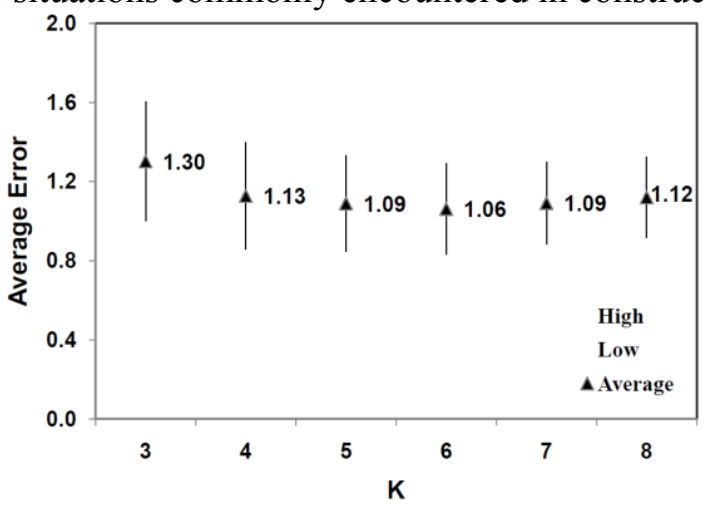

Figure 2: Average localization errors and its $95 \%$ confidence level interval for different $k$ (no car)

We tried to determine how the localization has changed qualitatively and quantitatively. To this end, we used localization error vectors to express the localization error. We note that such error vectors are also possible to plot in the case when no obstacle is present, but we nevertheless try to understand if the inclusion of the obstacle has a tendency to distort the localization in certain locations (relevant to the obstacle) and in what direction and by what magnitude.

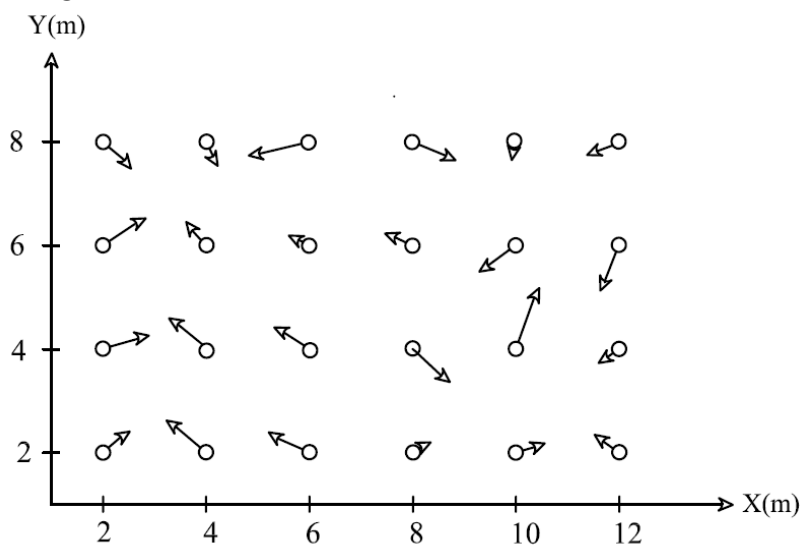

a) Without any obstacle

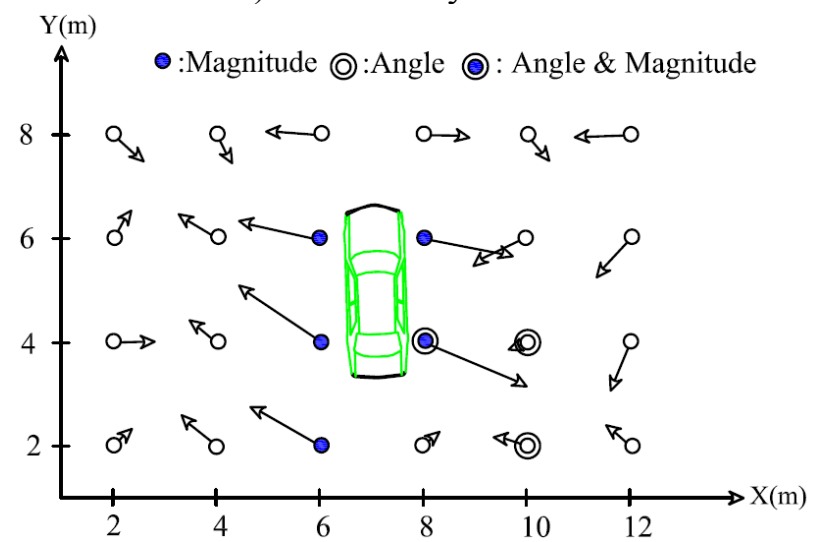

c) Car at middle side

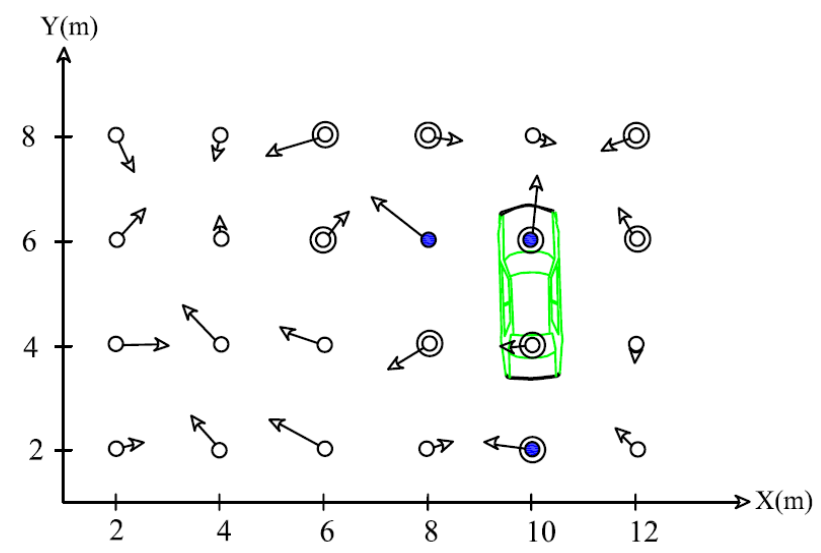

b) Car at right side

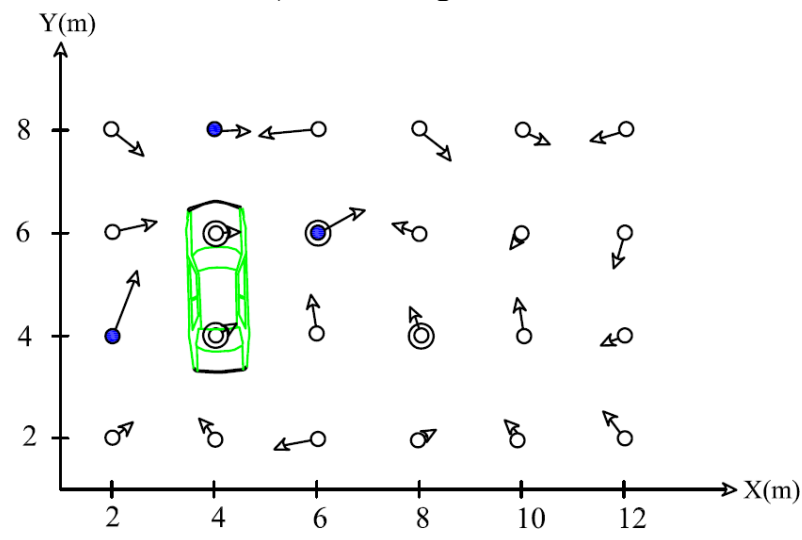

d) Car at left side

Figure 3. Localization error vector for different position of the car in the grid for $k=6$. 


\section{Soleimanifar, Lu, Nikolaidis and Lee}

The results are plotted in Figure 3 and they confirm our expectations to see more discrepancies close to the obstacle, albeit not in a systematic fashion. In Figure 3, for presentation's sake, arrows are scaled to half their actual magnitudes, and the tags whose location error vectors have been changed considerably either in error magnitude (more than $1 \mathrm{~m}$ ) or in terms of angle (more than $90^{\circ}$ ), are marked, respectively, by a blue dot and a circular ring (some points may exhibit both features). Noticeable differences in localization error magnitude and angle are shown with different icons.

The results from the experiments (Figure 4) indicate that the system is able to locate all the tags with the error magnitude between roughly $0.8 \mathrm{~m}$ and $1.9 \mathrm{~m}$ (with the approximate standard deviation of 1 meter for the case of car in the middle of the grid and 0.5 meter for the other three ones). It also demonstrates a metallic obstacle can change the environment and generally increases the localization error. The desired accuracy needed to locate mobile laborers in construction sites is 1.5 to 4 meters (Khoury and Kamat 2009; Torrent and Caldas 2009). Therefore, for the prototype system, a localization accuracy of less than $2 \mathrm{~m}$ is acceptable and the localization error we observed in the presence of a simple obstacle was marginally kept around the original localization error determined in the absence of the obstacle. The robust nature of this localization technique thus implies its potential for deployment in real-world dynamic construction sites, which are prone to constant changes as a result of the introduction of permanent as well as temporary obstacles. It is noteworthy that the limits of the positioning accuracy can be further enhanced by applying a finer grid setup in RSSI profiling or applying real time error correction algorithms. The second alternative is more cost effective and appealing to construction applications and will be pursued in the future.

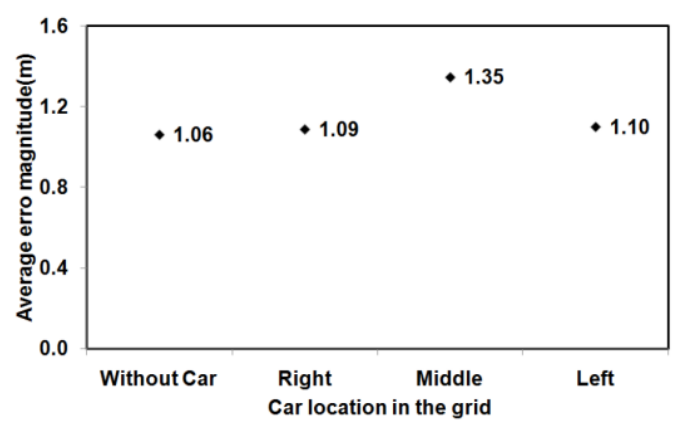

Figure 4. Average error magnitude using new profiling data for different car location in the grid for $k=6$

\section{RSSI CALIBRATION UTILIZING COMPLETE AND PARTIALLY RE-PROFILING}

The construction environment changes dynamically, which would mean that localization results might no longer be accurate in the (continuously) modified environment. To address this problem, two remedial approaches can be taken; (a) systematic analytical characterization of localization distortions created by modifications, and/or, (b) a means to perform re-profiling, assuming it is needed, to create more accurate localization results.

We note that option (a), apart from being a demanding task that entails some form of wireless propagation characteristic modeling, it would still not be sufficient because we would have to, first, identify that the environment has indeed changed before applying any analytical/computational localization correction model. If this requires re-measuring signal strengths (every so often) then it leads to effectively a form of option (b). Hence option (b) is an unavoidable step, even if it does not imply the re-measuring/resampling of all points in the space of interest. Re-profiling has merit of cancelling the effects of changes in the environment. We conducted the following experiment: in each test bed, each time an automobile was introduced at any of the three locations, new reference point measurements were collected and used to localize the tags. Figure 4 show the localization in the modified environments by using the previous profile data, while Figure 5 shows the localization accuracy using the new profile data. Both are able to 
result in the similar accuracy (Figure 4). The standard deviations are $0.47 \mathrm{~m}$ for car on the right, $0.71 \mathrm{~m}$ for car in the middle and $0.66 \mathrm{~m}$ for car on the left side of the monitored area.

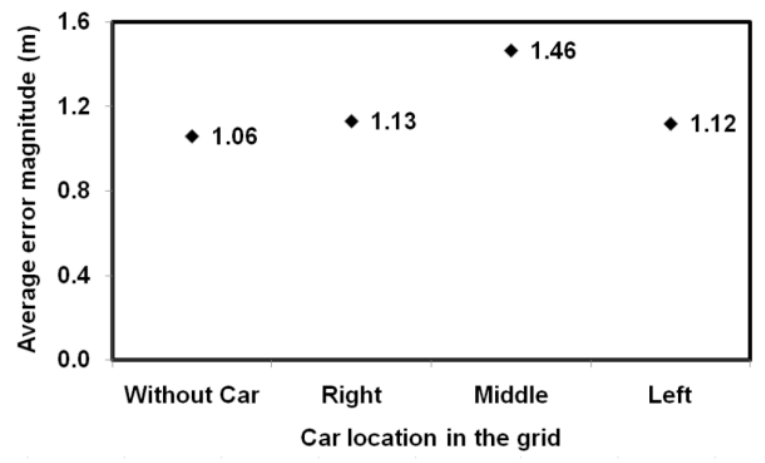

Figure 5. Average error magnitude for different car location in the grid for $k=6$

Of course the suggestion for re-profiling an entire area is quite demanding. To this end, we would like to "stage" the re-profiling task in two steps: (i) to understand what observations could trigger the reprofiling so no unnecessary re-profiling is performed and (ii) to determine a possible small subset of locations that, upon being re-profiled, result in improved localization. In other words, we imply a semiautomatic process whereby the task (i) is performed automatically and then a set of points to-be-reprofiled is prepared (again automatically) and provided to either human operators to conduct the task (ii) or, even, with an operator in the loop, choices for points to re-profile are given and acted upon.

If the localization pegs are part of a fixed (or rarely changing) infrastructure, then preference could be given to using them as profile points as well (we will explore this idea next), with the advantage that the re-profiling for those particular points can be performed without on-site human intervention (as opposed to re-profiling points in a construction area).

To address point (i), as previously seen, a significant increase of magnitude and angle change in localization error results from introducing a new obstacle to the environment. In this case, if it goes beyond a certain threshold (in our experiments the thresholds were $1 \mathrm{~m}$ and $90^{\circ}$ ), re-profiling can be necessary. To perform task (ii) in an automatic way, we treat the pegs as profiled points (we know their exact location). We identified the one peg which showed the most significant localization error when it was found (according to criterion (i)) after an obstacle was introduced. This operation is captured in Figure 6. Note that we are not done at this point because the location of the peg, which was identified as "most distorted" localization was based on $k$ measurements that could be all over the area of interest. At that point, we dispatched a human operator to re-profile the $k$ points involved. We also compared the results of this partial re-profiling to the results of a complete re-profiling.
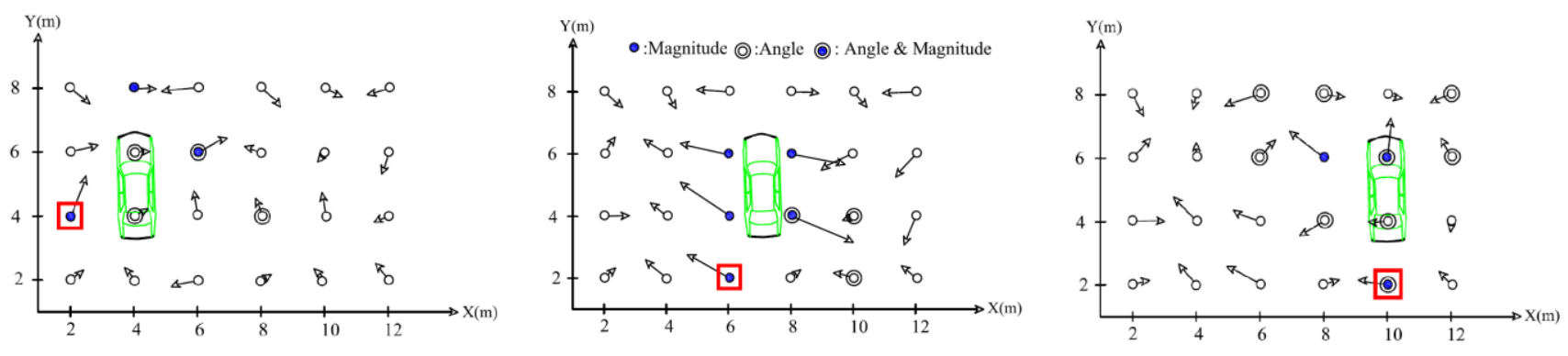

Figure 6. Selected co-located tags to be re-profiled

Table 1 shows that localization average error magnitude utilizing a number of partially re-profiled reference points (6) would result in the same improvement as that of complete re-profiling. These empiri- 
cal results suggest that the proposed approach can be used to monitor engineered components and construction resources on a typical dynamic job site with changing locations of obstacles. With less effort in performing partially re-profiling, the effect of obstacles on the job sites can be controlled and the accuracy can be maintained on a certain level.

Table 1: Localization average error magnitude using a) original profiling samples in absence of obstacle

b) partially and complete re-profiled reference points

\begin{tabular}{cccc}
\hline Car position & \begin{tabular}{c} 
Using Orig- \\
inal refer- \\
\cline { 3 - 4 } ence points
\end{tabular} & $\begin{array}{c}\text { Using re-profiled reference } \\
\text { points }\end{array}$ \\
\cline { 3 - 4 } & 1.13 & Partial(6) & Complete(25) \\
\hline Car at Right & 1.12 & 1.08 & 1.09 \\
Car at Left & 1.46 & 1.4 & 1.1 \\
Car at Middle & 1.24 & 1.19 & 1.35 \\
Average & & & \\
\hline
\end{tabular}

\section{CONCLUSION}

The presented study introduces a new framework for automating the identification and localization of construction resources on indoor or partially covered construction sites. In this approach a positioning methodology associated with wireless sensor networks was used to facilitate data collection and pattern recognition. Indoor experiments assessed the feasibility of this potentially automated methodology in a realistic construction setting. The localization approach resulted in good estimated locations with accuracies ranging from $1 \mathrm{~m}$ to $2 \mathrm{~m}$. Moreover, the localization accuracy in the presence of obstacles remains comparable to that achieved prior to the introduction of obstacles. In addition, it was found that With less effort in performing partially re-profiling, the effect of obstacles on the job sites can be controlled and the accuracy can be maintained on a certain level.. In comparison with the other technologies used for resource tracking in indoor or partially covered construction environments, the proposed system has the advantages in terms of networking flexibility, ease of deployment and extendibility, low cost, and the capacity to operate efficiently under dynamic and harsh conditions. The robust nature of this localization technique thus implies its potential for deployment in real-world dynamic construction sites. Those settings by nature are prone to constant changes as a result of the introduction of permanent as well as of temporary obstacles. It is noteworthy that the limits of the positioning accuracy can be further enhanced by applying a finer grid setup in RSSI profiling or applying real time error correction algorithms. The second alternative is more cost effective and appealing to construction applications and will be pursued in the future.

\section{ACKNOWLEDGEMENTS}

The authors would like to acknowledge the work of Dr. Israat T. Haque and Dr. Pawel Gburzynski for the development of the tools and their support for this project.

\section{REFERENCES}

Cho, C.Y., S. Kwon, T.H. Shin, S. Chin and Y.S. Kim. 2011. "A development of next generation intelligent construction liftcar toolkit for vertical material movement management", J. Automation in Construction, 20:14-27.

D. Niculescu and B. Nath. 2001. "Ad hoc positioning system (APS)", In International Conference on Global Communication (GLOBECOM),2926-2931, Ottawa, Canada. 
Haque, I.T., I. Nikolaidis, and P. Gburzynski. 2009. "A scheme for indoor localization through RF profiling", Proceedings of ICC/SyCoLo, Dresden, Germany.

He, T., C. Huang, B.M. Blum, J.A. Stankovic, and T. Abdelzaher. 2003. "Range-free localization schemes for large scale sensor networks", Proceedings of the 9th annual international conference on Mobile computing and networking, 81-95, New York, NY, USA .

Jang, W.S., and M.J. Skibniewski. 2009. "Embedded System or Construction Asset Tracking Combining Radio and Ultrasound Signals", Journal of Computing in Civil Engineering, ASCE, 23(4): 221-229.

Khoury, H.M., and V.R. Kamat. 2009. "Evaluation of position tracking technologies for user localization in indoor construction environments", J. Automation in Construction, 18: 444-457.

Kim, C., H. Kim, J. Ryu, and C. Kim. 2010. "Ubiquitous Sensor Network for Construction Material Monitoring", J Construction Eng. and Management, 158-165.

Lu, M., W. Chen, X. Shen, H.C. Lam and J. Liu. 2007. "Positioning and tracking construction vehicles in highly dense urban areas and building construction sites", J. Automation in Construction,16: 647656.

Luo, X., W.J. O’Brien, C.L. Julien. 2010. “Comparative evaluation of Received Signal-Strength Index (RSSI) based indoor localization techniques for construction job sites", J. Advanced Engineering Information, 1-9.

Lymberopoulos, D., Q. Lindsey, and A. Savvides. "An Empirical Analysis of Radio Signal Strength Variability in IEEE 802.15.4 Network Using Monopole Antennas", Technical Report 050501,Embedded Networks and Applications Lab (ENALAB), Yale University, CT.

Pradhan, A., E. Ergen, and B. Akinci. 2009. "Technological Assessment of Radio Frequency Identification Technology for Indoor Localization", Automation in Construction, 23(4):230-238 .

Shen, X., W. Chen, and M. Lu. 2008. "Wireless Sensor Networks for Resources Tracking at Building Construction Sites". Tsinghua Sci. and Tech.,13:78-83.

Shin,T.H., S. Chin, S.W. Yoon, and S.W. Kwon. 2011. "A service-oriented integrated information framework for RFID/WSN-based intelligent construction supply chain management", J. Automation in Construction.

Skibniewski, M.J. and W.S. Jang. 2009.” Simulation of Accuracy Performance for Wireless Sensor-Based Construction Asset Tracking", Computer-Aided Civil and Infrastructure Eng., 24: 335-345.

Teizer, J. 2008. "Ultrawideband for Automated Real-Time Three-Dimensional Location Sensing for Workforce, Equipment, and Material Positioning and Tracking" J. the Transportation Research Board, 56-64.

Torrent, D.G., and C.H. Caldas. 2009. "Methodology for Automating the Identification and Localization of Construction Components on Industrial Projects", J. Computing in Civil Eng, 23:1(3):3-13.

Zhang, D., F. Xia, , Z. Yang, , L. Yao and W. Zhao. 2010. " Localization Technologies for Indoor Human Tracking", 50th International Conference on Future Information Technology, 1- 6. Busan, Korea.

\section{AUTHOR BIOGRAPHIES}

MEIMANAT SOLEIMANIFAR is a M.Sc. student in Department of Civil and Environmental Engineering, Construction Engineering and Management Program at the University of Alberta. She received her B.Sc. in Electrical Engineering from the University of Yazd, Iran, in 2006. Her research interest is on management of construction dynamics with the help of resource positioning and automation in real-time to support decision-making. Her e-mail address is meimanat@ualberta.ca.

MING LU is an Associate Professor in the Department of Civil \& Environmental Engineering at the University of Alberta. He has been committed to achieving excellence in research and teaching in areas of construction engineering and project management. He had worked as site engineer and project manager for three years in the construction field prior to beginning his pursuit of Ph.D in Construction Engineering and Management at the University of Alberta in Aug. 1997. In Nov. 2000, Dr. Lu joined the Department 


\section{Soleimanifar, Lu, Nikolaidis and Lee}

of Civil and Structural Engineering of the Hong Kong Polytechnic University. In September 2010, Dr. Lu assumed a position of Associate Professor at the University of Alberta, Canada. His research interests are construction surveying and automation; operations simulation and scheduling in construction. His email addressismlu6@ualberta.ca.

IOANIS NIKOLAIDIS is an Professor with the Computing Science Department at the University of Alberta. He received his B.Sc. from the University of Patras, Greece, in 1989 and his M.Sc. and Ph.D. from the Computer Science Department at Georgia Tech in 1991 and 1994, respectively. During 1995-96 he worked for the European Computer-Industry Research Center in Munich, Germany. His research interests include wireless sensor networks and performance evaluation of computer network protocols. His e-mail address is nikolaidis@ualberta.ca.

SANGHYUN LEE is an Assistant Professor at the University of Michigan. Before joining the faculty there, Dr. Lee worked as an Assistant Professor at the University of Alberta, Canada, from 2007 to 2010. He earned Ph.D. in Construction Management and Information Technology from MIT, and worked at CRA International as a consultant and participated in numerous international construction projects while teaching Project Management at MIT (2006-2007). Dr. Lee's main research interest is the understanding and management of construction dynamics through the design and development of mechanisms, models, and systems that enhance proactive management of mega construction projects. His e-mail is shdpm@umich.edu. 